\title{
«Les mauvais médecins ne veulent pas de certificats» [1]
}

Jean-Luc Boss

Correspondance:

Dr Jean-Luc Boss

Médecin spécialiste en psychiatrie

et psychothérapie FMH

Rue du Mont-Blanc 21

CH-1201 Genève

Tél. 0227388828

jeanboss@hin.ch
Affirmation péremptoire et quasiment définitive de K. Hess, médecin, économiste ... et membre de la fondation EQUAM (organe de certification reconnu depuis 2003 par le Service d'accréditation suisse SAS du SECO), dont la croissance économique est apparemment tout à fait réjouissante, soutenue qu'elle est par une «liste de sponsors connus issus du milieu des assurances maladie et accident, [...] et de l'industrie pharmaceutique».

Au-delà des intérêts financiers bien compréhensibles de toute une série d'agents de certification, coaches, consultants et autres auditeurs qui ont bien vite compris l'extraordinaire potentiel lucratif de ce commerce qualifié - en raison de leur marges bénéficiaires exceptionnelles de 20 à $30 \%$ - de «machine à faire du fric» par le journal Tages-Anzeiger [2], il me semble indispensable de s'interroger, en tant que soignants, sur la véritable plus-value apportée par les diverses certifications «qualité», aux soins eux-mêmes.

Concernant les traitements hospitaliers, une grande étude publiée en 2005 dans le JAMA [3], a comparé la prise en charge de cinq pathologies nant parfois directement le revenu du praticien, ce qui équivaut au «contrôle de la qualité via le porte-monnaie» selon les termes de la Tribune Médicale [5].

Le système britannique du «New Contract» se base sur un indice de qualité (QOF: Quality Outcome Framework) fondé sur un système de points et détermine $21 \%$ (!) du revenu des médecins.

Par exemple, pour obtenir ces précieux points, le médecin traitant de patients coronariens doit s'assurer qu'au moins $60 \%$ de ceux-ci ont un cholestérol inférieur à $5 \mathrm{mmol} / \mathrm{l}, 50 \%$ au moins sont traités par un bêtabloquant, 70\% des insuffisants cardiaques reçoivent un IEC ou un sartans et qu'au moins $85 \%$ sont vaccinés contre la grippe. D'où l'apparition d'une nouvelle sorte de collaborateurs médicaux, spécialisés dans la «traque» des patients ne rapportant pas assez de points et leur rappel (convocation?) en consultation afin de remédier à cette situation financièrement inopportune pour le praticien.

Le risque d'un fonctionnement médical se basant sur une rétribution de type «pay-for-performance» [6], étant évidemment de voir les pra-

\section{Quasi parfaite égalité entre 199 hôpitaux «certifiés»}

\section{et 142 institutions «non certifiées» concernant la prise en charge de cinq pathologies classiques}

classiques (fibrillation auriculaire, infarctus myocardique, insuffisance cardiaque, pneumonie et accident cérébrovasculaire) par 199 hôpitaux «certifiés» ou par 142 institutions «non certifiées». Le commentaire de Alain Junod [4] à propos de ces résultats était particulièrement significatif: non seulement on pouvait conclure à la «quasi parfaite égalité entre les deux groupes», mais de plus, le risque existe, bien réel, d'un désengagement des soignants de leur sens des responsabilités et de leur motivation, suite aux «innombrables procédures, rappels et courriels» dus aux contrôles délégués à des organismes extérieurs aux soins.

La médecine ambulatoire peut également faire l'objet d'une évaluation qualité, détermi- ticiens s'intéresser de plus en plus aux indicateurs de santé (quelque soit leur pertinence vis-à-vis du cas particulier d'un patient ...) au détriment d'une prise en soins globale et réfléchie.

Il ne faut bien évidemment pas ignorer certains critères para-cliniques bien définis et mesurables (glycémie des diabétiques) ou certaines pratiques thérapeutiques (antibiothérapie des pneumonies ou méningites) qui sont à la base de recommandations pratiques possédant le «haut niveau de preuve» requis pour définir une qualité de soins réelle, et dont le non-respect entraîne une augmentation de la morbidité et de la mortalité.

Mais cette manière de procéder n'est-elle pas inhérente à toute bonne attitude médicale 
(Mr Jourdain faisait de la prose sans le savoir ...) et doit-elle vraiment être codifiée et quantifiée, avec le risque déjà évoqué d'une attention excessive donnée aux indicateurs plutôt qu'au patient?

Si la question mérite d'être posée pour la médecine somatique, elle est d'autant plus importante en psychiatrie. Certes, comme le souligne

\section{Si la question mérite d'être posée pour la médecine somatique, elle est d'autant plus importante en psychiatrie}

Monika Raidl-Dengler (Dr en économie) dans le dernier bulletin Psy et Psy [7], on peut bien évidemment quantifier le nombre de pièces du cabinet, la surface de la salle d'attente, la présence de toilette et celle d'un ascenseur ... mais est-il raisonnable d'imaginer que l'on a vraiment défini ainsi la «situation et [les] aménagements optimaux du cabinet pour offrir une psychothérapie de haut niveau»?

Le problème du diagnostic en psychiatrie met cruellement en évidence l'absence de tests paracliniques fiables et utilisables dans la pratique quotidienne. Il s'ensuit que les différentes classifications diagnostiques (CIM, DSM) ne sont pas forcément concordantes et qu'une simple variation quantitative des critères retenus fait varier chothérapie d'un type particulier (analytique, cognitive ou systémique pour ne citer que les principales écoles) en guise de traitement de pathologies aussi mal définissables, avec en prime le risque de raviver les éternelles «guerres de religions» thérapeutiques?

Quand aux thérapies biologiques, si leur efficacité dans la pratique de tous les jours est indéniable, les grandes méta-analyses peinent parfois à établir leur bénéfices réels [8], en raison notamment de critères d'efficacité purement arbitraires fixés par des organismes (à l'exemple du NICE) officiellement dévolus à la détermination de «l'excellence clinique».

Ce qu'a pu démontrer l'étude CATIE [9] dans laquelle plus de 1000 patients souffrant de schizophrénie chronique voyaient leur traitement habituel subitement remplacé «à l'aveugle» et dont la situation se péjorait dans près de $75 \%$ (!) des cas - n'est pas la supériorité de tel neuroleptique sur un autre mais la nécessité absolue d'un traitement individualisé, échappant à toute directive contraignante.

Notre confrère le Dr Beat Künzi, directeur médical de swisspep, semble convaincu de la fiabilité des patients psychiatriques concernant l'évaluation de leur satisfaction [10], allant même jusqu'à juger «dangereux» (!) d'en douter ...

Sans vouloir nier l'importance de la satisfaction de tout patient il faut cependant garder à l'esprit que le résultat du questionnement de cette

\section{II reste l'espoir de ne pas voir se développer et se généraliser un système de qualification uniquement «parasitaire» c'est-à-dire sans réelle valeur ajoutée aux soins des patients}

incidence et prévalence du trouble (la prévalence des troubles du spectre bipolaire atteint 40\% [!] de la population zurichoise selon les critères élargis de certains experts).

Les échelles d'évaluation (Hamilton, Beck, MARDS, MMS, inventaire neuro-psychiatrique, etc.) peuvent s'avérer utiles pour évaluer de manière individuelle et ponctuelle certains aspect pathologiques, mais on ne saurait les relier aussi simplement à la qualité d'une prise en soins. Le risque étant bien évidemment un résultat d'évaluation fortement biaisé, en fonction des avantages financiers induits par certains scores, dans le cas d'un système de type «pay-for-performance».

Sur le plan thérapeutique, c'est encore moins clair ... Peut-on en effet réellement indiquer (imposer?) avec suffisamment de certitude, une psy- donnée dépend en grande partie du moment (et de la manière) de poser ces fameuses questions. La variabilité de l'avis étant quasiment toujours fonction de l'état psychologique du patient, par définition sujet à modification, parfois même très rapide selon la pathologie en cause. Il s'agit donc d'un argument de certification particulièrement volatil et pour le moins fragile.

En outre, de manière générale, le «niveau de satisfaction du secteur psychiatrique se situe $15 \%$ plus bas que les autres» comme le rapporte Olivier Peters, directeur des Hospices CHUV du canton de Vaud [11].

Pour finir ce très (trop) rapide aperçu de la «pratique psychiatrique en voie de certification», mentionnons encore les établissements médicaux-sociaux (EMS), dont la majorité doit faire 
face à l'augmentation continue des affections neuro-psychiatriques, sur fond de polymorbidité de plus en plus lourde.

Des ressources très considérables ont été investies afin d'obtenir diverses certifications, dans un contexte budgétaire pourtant difficile et nécessitant de faire des choix. Peut-être ces attestations représentent-elles le sésame indispensable à l'obtention des subventions étatiques, mais en tout état de cause, les certificats ostensiblement exhibés à l'entrée des établissements ne peuvent être reliés en aucune façon à la pertinence des soins, que seuls la formation, l'encadrement et l'engagement des soignants peuvent assurer.

En guise de très provisoire conclusion, il reste l'espoir de ne pas voir se développer et se généraliser un système de qualification uniquement «parasitaire» c'est à dire sans réelle valeur ajoutée aux soins des patients. Pour cela, la formation continue des médecins, l'encadrement motivant des soignants (en EMS par exemple) et le partage des informations entre eux (les réseaux inofficiels existent depuis bien longtemps!) semblent à même d'assurer une qualité véritable des soins, en utilisant des échelles d'évaluation et des paramètres biologiques à bon escient, et non de manière systématiquement contraignante ni à visée rémunératrice.

A propos de la satisfaction des patients, l'indice le plus fiable la qualifiant réellement ne serait-il pas la continuité du traitement chez leur thérapeute? Dans le même ordre d'idée, le véritable danger ne résiderait-il pas dans une impossibilité de changer en cas de mécontentement avéré et non surmontable? Ce point de vue très éloigné de la démarche qualité officiellement proposée met en évidence une fois de plus l'importance du libre choix du médecin que la votation

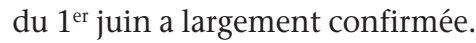

On peut cependant légitimement craindre une évolution de la politique de santé vers l'imposition généralisée d'une sorte de «taxe (rançon?) qualité» imposée à tout le corps médical, débouchant sur une certification officielle dont la valeur informative serait aussi crédible qu'une visite du site www.okdoc.ch.

Le risque existe aussi d'une vision purement commerciale et comptable de la médecine, pilotée par des indices du type ANOVA ou, telle que celle prônée par le directeur du groupe des cliniques Hirslanden, Robert Bider [12], considérant le «patient devenu client» comme un simple consommateur de services, les soignants comme du personnel dont la «formation est trop longue» et donc trop cher, adepte d'une libéralisation totale du marché et s'appuyant pour ce faire sur un efficace «travail de lobby dans les cantons et au niveau national ...».

«Aucune avancée n'est plus difficile que le retour à la raison»

B. Brecht

\section{Références}

1 SENSO; 2006.

2 Le Temps, 12.5.2006.

3 Snyder C, Anderson G. Do quality improvement organizations improve the quality of hospital care for Medicare beneficiaries? JAMA 2005; 293: 2900-7.

4 Junod AF. Le contrôle de qualité des soins est-il efficace? Rev Méd Suisse. 2005;1:2755.

5 Tribune médicale, 17.3.2006

6 Heuss LT. P 4 P. Bull Méd Suisses. 2006;87(38):1631.

7 Raidl-Dengler M. N'ayons pas peur de la gestion de qualité. Psy \& Psy. 2008;(1):10-1.

8 Kiefer B. Antidépresseurs: le doute. Rev Méd Suisse. 2008;4(154):1072.

9 Lieberman JA, Stroup TS, McEvoy JP, Swartz MS, Rosenheck RA, Perkins DO, et al. Effectiveness of antipsychotic drugs in patients with chronic schizophrenia. N Eng J Med. 2005;353:1209-23.

10 Attinger Andreoli Y. «La qualité dans le domaine médical est un phénomène très complexe». Interview du Dr Beat Künzi. Psy \& Psy 2008;(1):17-20.

11 Le Temps, 10.6.2008.

12 Le Temps, 14.6.2008. 